\title{
Uso de suelo en laderas del sector sur de la Comuna de Lonquimay (Chile) y sus características estructurales
}

\author{
Vial-Alarcón, M. ${ }^{+*}$
}

\section{RESUMEN}

Se determinaron indicadores de calidad física de suelo en una ladera del sur de la comuna de Lonquimay ( $38^{\circ} 37^{\prime} 38^{\prime \prime}$ Latitud Sur; $71^{\circ} 05^{\prime} 26^{\prime \prime}$ Longitud Oeste) como unidad fisiográfica uniforme sometida a 3 usos: Bosque bajo pastoreo (BP) compuesto por Ñirre (Nothofagus antárctica), Pradera sembrada (PS) de Alfalfa (Medicago sativa) y Pradera degradada (PD) (Acaena sp., Chusquea sp., Festuca sp., Stipa sp.). Son suelos Franco Arenosos (media de 60,6\% arena, 31,3\% limo y 8,1\% de arcilla) sometidos a pastoreo caprino. Presenta una porosidad alta ( $>52 \%)$. Existieron diferencias en el contenido de $\mathrm{MO}$ en la secuencia $\mathrm{BP}>\mathrm{PS}>\mathrm{PD}$. Existieron diferencias en Macroagregados $(\mathrm{PD}>\mathrm{BP}>\mathrm{PS}$ ) y Microagregados ( $\mathrm{PS}>\mathrm{PD}>\mathrm{BP}$ ). Al medir el Diámetro Peso Medio DPM (mm), como indicador de estabilidad de agregados, se esperaba un mayor valor BP por la presencia de materia orgánica lo cual no ocurrió; todos los valores de DPM fueron $<0,2 \mathrm{~mm}$, considerados como agregados inestables. Se concluye que los suelos estudiados presentan condiciones generalizadas de degradación estructural, independiente a su uso y profundidad; esto ocurre debido al efecto aditivo de una serie de factores de degradación tales como un bajo contenido de materia orgánica, erosión, escasa estructura de los suelos, baja estabilidad de agregados y pastoreo excesivo que no permite recuperar la cubierta vegetal; a lo cual se suman condiciones climáticas con bajas temperaturas que ralentizan la dinámica biológica.

Palabras clave: Uso de suelo, pastoreo, estabilidad de agregados, degradación, erosión.

1.- INIA Centro Regional de Investigación Carillanca, kilómetro 10 camino Cajón Vilcún, Región de La Araucanía, Chile. *Autor por correspondencia:manuel.vial@inia.cl

Manuscrito recibido el 15 de marzo de 2021 y aceptado para su publicación el 28 de septiembre de 2021.

Vial-Alarcón, M. Uso de suelo en laderas del sector sur de la Comuna de Lonquimay (Chile) y sus características estructurales. FAVE - Ciencias Agrarias 21 (1): 99-112 - CC BY-NC-SA 4.0 


\begin{abstract}
Land use in the hills of the southern sector of the Lonquimay commune (Chile) and its structural characteristics.

Soil physical quality indicators were determined on a hillside in the south of Lonquimay commune (38³7’38" South Latitude; $71^{\circ} 05^{\prime} 26^{\prime \prime}$ 'West Longitude) as a uniform physiographic unit subjected to 3 uses: Nirre (Nothofagus antarctica) grazed forest (BP), Alfalfa (Medicago sativa) sown pasture (PS) and degraded pasture (PD) (Acaena sp., Chusquea sp., Festuca sp., Stipa sp.). The soils are sandy loam (average $60.6 \%$ sand, $31.3 \%$ silt and $8.1 \%$ clay) subjected to goat grazing. They have a high porosity $(>52 \%)$. There were differences in $\mathrm{OM}$ content in the sequence $\mathrm{BP}>\mathrm{PS}>\mathrm{PD}$. There were differences in macroaggregates $(\mathrm{PD}>\mathrm{BP}>\mathrm{PS}$ ) and microaggregates $(\mathrm{PS}>\mathrm{PD}>\mathrm{BP})$. When measuring the Mean Weight Diameter MWD ( $\mathrm{mm})$, as an indicator of aggregate stability, a higher BP value was expected due to the presence of organic matter, which did not occur; all MWD values were $<0.2 \mathrm{~mm}$, considered as unstable aggregates. It is concluded that the soils studied show generalised conditions of structural degradation, independent of their use and depth; this occurs due to the additive effect of a series of degradation factors such as low organic matter content, erosion, poor soil structure, low aggregate stability and overgrazing that does not allow for the recovery of vegetation cover; to which are added climatic conditions with low temperatures that slow down biological dynamics.
\end{abstract}

Key words: Land use, grazing, aggregate stability, degradation, erosion.

\section{INTRODUCCIÓN}

Escenarios geográficos simulados indican que en 50 a 70 años existirán fuertes modificaciones climáticas centradas en variabilidades en regímenes de temperatura y precipitaciones en cantidad e intensidad. Informes en base a escenarios considerados por el IPCC señalan una disminución de la precipitación entre 5 y $15 \%$, para las latitudes $27^{\circ} \mathrm{S}$ a $45^{\circ} \mathrm{S}$, alzas de temperaturas medias y variación ascendente de la isoterma cero entre 350 a 500 m.s.n.m, siendo reemplazada las precipitaciones nivales por lluvia (Universidad de Chile, 2012; FIA, 2017).

La comuna de Lonquimay, ubicada en la Región de La Araucanía de Chile, tiene una superficie de $3914 \mathrm{~km}^{2}$ y basa su economía en la ganadería extensiva y explotación forestal; concentra el $90 \%$ de los caprinos, $35 \%$ caballares, $16 \%$ bovinos y $31 \%$ de ovinos de la región. El uso ganadero se basa en la dinámica invernada-veranada, donde son utilizadas las praderas ubicadas en sectores bajos y terrazas fluviales durante el invierno (invernada) y desde el mes de diciembre-abril son utilizadas las praderas de sectores altos, donde se ha retirado la nieve (veranada). La pluviometría anual varía entre los $1120 \mathrm{~mm}$ a $2000 \mathrm{~mm}$ según la fisiografía. Climáticamente, el sector de Pehuenco es calificado en una macro zona como templado frío con régimen de humedad húmedo con tendencia mediterránea (Cfcfs) y en específico como templado cálido mesotermal con régimen de humedad sub húmedo-húmedo (Csb1Shh) con una 
precipitación media anual de hasta 2470 $\mathrm{mm}$ en la macrozona, sin periodo libre de heladas, con 3 meses de temperaturas favorables para el desarrollo de la actividad vegetativa, 9 meses de receso vegetativo y un periodo seco de 2 meses (FIA, 2017).

El objetivo del presente trabajo es determinar la existencia de diferencias entre distintos usos de suelo respecto a indicadores de calidad física y en particular la estabilidad estructural en un sector de ladera (Pehuenco) bajo uso en el sistema tradicional de pastoreo caprino extensivo y sin control de carga animal, entre la cota 850 a 1350 m.s.n.m., ubicada en la franja altitudinal sensible de ser afectada por la variabilidad y eventos climáticos adversos proyectados.

\section{MATERIALES Y MÉTODOS}

\section{Caracterización de los distintos usos de suelo}

Se estudiaron tres usos de suelo con fines agropecuarios orientados a ganadería caprina en un lugar representativo de ladera en el sector Pehuenco (38 $37^{\prime} 38^{\prime \prime}$ Lat. S.; $71^{\circ} 05^{\prime} 26^{\prime \prime}$ Long. O.). El uso del suelo (U) se consideró como tratamiento. El muestreo se realizó en tres profundidades $(\mathrm{P})$ (sub-tratamientos) en estratos de 0-5, 5-15 y $15-25 \mathrm{~cm}$ respectivamente, en una altitud considerada como punto medio de 1190 m.s.n.m. (Figura 1). Los usos de suelo y sus características son las siguientes:

* Bosque bajo pastoreo (BP): corresponde a una formación boscosa de Ñirre
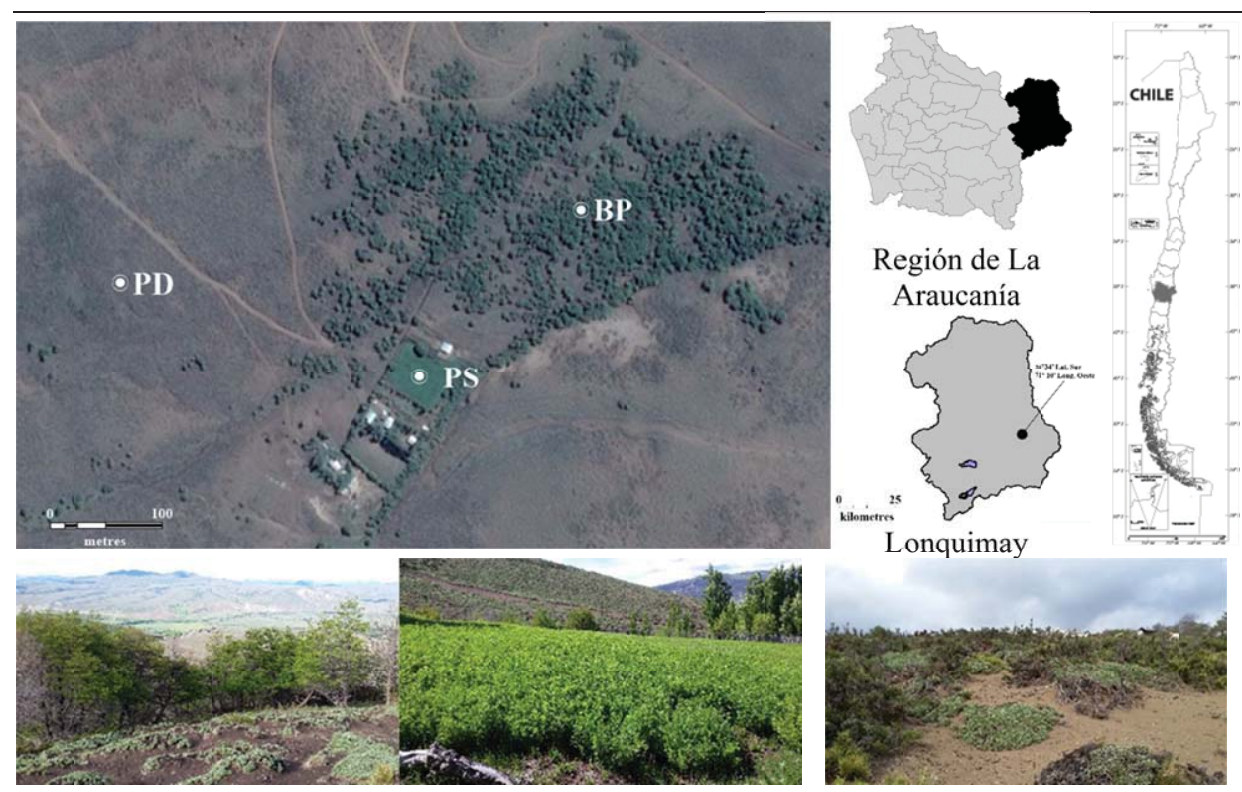

Bosque bajo pastoreo (BP)

Pradera sembrada (PS)

Pradera degradada (PD)

Figura 1. Ubicación y usos de suelo en ladera del sector Pehuenco.

Figure 1. Location and land use in the Pehuenco sector. 
(Nothofagus antarctica (G. Forst.) Oerst.), caducifolia en baja densidad (3-4 individuos en $10 \mathrm{~m}^{2}$ ) altamente intervenido por pastoreo de caprinos bajo el dosel.

* Pradera sembrada (PS): corresponde a alfalfa (Medicago sativa) mayor a 3 años, plantas en buen estado, ubicada en un sector de pendiente menor a $15 \%$. El espacio no es destinado al pastoreo, solo para corte y almacenamiento como forraje invernal.

* Pradera degradada (PD): corresponde a una pradera natural, altamente perturbada y degradada por el sobrepastoreo, con la formación de espacios sin vegetación (lunares de arena). Se encuentran especies tales como: Acaena splendens, Acaena sp., Chusquea sp., Festuca sp., Stipa sp., y arbustos dispersos como Colletia spinosa y Discaria chacaye (Hauenstein et al., 2003; Fuentes-Ramírez et al., 2011).

\section{Análisis}

El contenido de materia orgánica (MO\%) se determinó con el método de oxidación con dicromato de potasio de acuerdo con Sadzawka et al. (2006). La clase textural se determinó mediante el método de Bouyoucos (Sandoval et al., 2012) y densidad aparente $\left(\mathrm{Da} \mathrm{g} \mathrm{cm}^{-3}\right)$ mediante método de cilindro. El \% humedad a capacidad de campo C.C. y punto de marchitez permanente P.M.P (33 y $1500 \mathrm{~atm}$. respectivamente) se determinó con el método de placas de presión (Sandoval et al., 2012). La porosidad total $(\mathrm{Pt})$ determino según la formula indicada por Hao et al. (2019) considerando como referencia para suelos minerales el valor de 2,65 de densidad real Dr $\left(\mathrm{g} \mathrm{cm}^{-3}\right)$ (Schlatter et al., 2003; Blake, 2008). A partir de Pt (\%) se determinó: Macroporos (Ma\%), Microporos $(\mathrm{Mi} \%)$ y Mesoporos $(\mathrm{Me} \%)$ (Salas y Cabalceta, 2009) de acuerdo a las siguientes fórmulas:
* Porosidad total $(\mathrm{Pt} \%)=[1-(\mathrm{Da}(\mathrm{g}$ $\left.\left.\mathrm{cm}^{-3}\right) / \operatorname{Dr}\left(\mathrm{g} \mathrm{cm}^{-3}\right)\right]$

* Macroporos $(\mathrm{Ma} \%)=\mathrm{Pt}(\%)-[\%$ C.C. $\left.* \mathrm{Da}\left(\mathrm{g} \mathrm{cm}^{-3}\right)\right]=[\varnothing>50 \mu \mathrm{m}]$

* Mesoporos $(\mathrm{Me} \%)=[\%$ C.C. $*$ Da $(\mathrm{g}$ $\left.\left.\mathrm{cm}^{-3}\right)\right]-\left[\%\right.$ P.M.P. $\left.* \mathrm{Da}\left(\mathrm{g} \mathrm{cm}^{-3}\right)\right]=[\varnothing 50 \mu \mathrm{m}-$ $0,2 \mu \mathrm{m}]$

* Microporos $(\mathrm{Mi} \%)=[\%$ P.M.P. $* \mathrm{Da}(\mathrm{g}$ $\left.\left.\mathrm{cm}^{-3}\right)\right]=[\varnothing \leq 0,2 \mu \mathrm{m}]$

El porcentaje de agregación se determinó mediante el método de tamizado en húmedo, donde una muestra de suelo (100 g) es colocada sobre juego de tamices sobrepuestos de distintos diámetros (2-1 mm; 1-0,5 mm; $0,5-0,25 \mathrm{~mm} ; 0,25-0,1 \mathrm{~mm} ; 0,1-0,05 \mathrm{~mm}$ y material $\leq 0,05 \mathrm{~mm}$ ) y es sometida a una columna de agua en oscilaciones verticales (25 osc./min, durante $10 \mathrm{~min}$ ). Los agregados son retenidos y clasificados en Macroagregados $\left(\sum \geq 0,25 \mathrm{~mm} \varnothing\right)$ y Microagregados $\left(\sum<0,25 \mathrm{~mm} \varnothing\right)$. La estabilidad de agregados se determinó utilizando como indicador el Diámetro Peso Medio (DPM mm) (Kemper y Rosenau, 1986), y el Índice de Perdida (IP $\mathrm{g} \mathrm{kg}^{-1}$ ) se determinó con el material no retenido por el tamiz $(\leq 0,05$ $\mathrm{mm}$ ) (Rodríguez et al., 2006; Sandoval et al., 2011; Vial y Sandoval, 2015).

Las fórmulas utilizadas son las siguientes:

$\mathrm{DPM}=\sum\left[\left(x i^{*} w i\right) / 100\right]$, donde $x i$ es el diámetro medio de cada fracción de tamaño $(\mathrm{mm})$ y wi es el peso de la muestra $(\mathrm{g})$ en esa fracción

$\mathrm{IP}=[(\mathrm{M}$ total suelo seco $-(\mathrm{MA}$ seco

+ MI seco)) $\left.\mathrm{g} \mathrm{kg}^{-1}\right]=$ material no retenido $(\leq 0,05 \mathrm{~mm})$

$\mathrm{M}$ total suelo seco $=100 \mathrm{~g}$ suelo

MA suelo seco = Macroagregados $\left(\sum \geq 0,25 \mathrm{~mm} \varnothing\right)$

MI suelo seco = Microagregados $\left(\sum<0,25 \mathrm{~mm} \varnothing\right)$ 


\section{Clasificación de suelos}

Se consideró como referencia la clasificación señalada por Luzio et al. (2013) como Dystrudepts (Orden Inceptisol) señalado como Asociación Lonquimay (LQM), y la realizada por Tosso (1985) como orden Entisol (Orthents, Psamments); ambos caracterizados por presentar arenas con granulometría variable y baja saturación de bases ocupando laderas de valles fluvioglaciales. En superficie los suelos son de textura franco arenosa de color pardo grisáceo (10YR 3/2) en seco y pardo grisáceo oscuro (10YR 2/2 a 10YR 3/1) en húmedo; en profundidad $(60 \mathrm{~cm})$ cambia a pardos gris (10YR 3/2) en seco a pardo oscuro $(7,5 \mathrm{YR}$ 2,5/2) en húmedo. El arraigamiento radicular es variable, superando los $60 \mathrm{~cm}$ y está limitado por un substrato no continuo compuesto por gravas de diverso tamaño (Tosso, 1985; Luzio et al., 2009; CIREN, 2010).

\section{Análisis estadístico}

El diseño fue completamente al azar, considerando el uso de suelo (U) como tratamiento bajo una unidad fisiográfica uniforme (ladera-sitio) y profundidades $(\mathrm{P})$ como sub-tratamientos. Se realizaron 3 repeticiones por tratamiento y sub-tratamiento. En cada variable se determinó la normalidad mediante la prueba Kolmogorov Smirnov y homogeneidad de las varianzas con la prueba de Levene; al no existir una distribución normal se aplicó la formula $(\mathrm{x}+0,5)^{1 / 2}$; la transformación en Arcoseno se utilizó en los valores expresados en porcentaje como las porosidades, texturas y humedad aprovechable. Se determinaron diferencias entre usos $(\mathrm{U})$ y profundidades (P), considerando significancias $\mathrm{p} \leq 0,05$ y $\mathrm{p} \leq 0,001$. Se determinó el Coeficiente de Variación C.V. $(\%)[(\sigma / \bar{x}) * 100]$ con el fin de conocer la variación de tratamiento y sub-tratamientos. Al ser significativas, las diferencias de medias fueron analizadas mediante Tukey considerando $\mathrm{p} \leq 0,05$.

\section{RESULTADOS Y DISCUSIÓN}

\section{Materia orgánica y textura}

Según la Tabla 1 existen diferencias significativas en el contenido de Materia Orgánica (MO) entre los distintos usos $(\mathrm{p}<0,05)$ en la secuencia $\mathrm{BP}>\mathrm{PS}>\mathrm{PD}$. Considerando el valor medio de $0-25 \mathrm{~cm}$, BP tuvo un contenido de MO 61\% sobre el contenido de PS y $142 \%$ sobre PD, esto atribuible al aporte constante de MO que realiza la formación boscosa (BP) al suelo. No existieron diferencias significativas $(p>0,05)$ en profundidad (U) ni interacción (UxP).

Existieron diferencias significativas $(p \leq 0,05)$ en el contenido de arena, limo y arcilla entre los distintos usos de suelo (U) e interacción significativa $(p \leq 0,05)$ entre uso de suelo y profundidad (UxP); la interacción se produce en el estrato superficial $(0-5 \mathrm{~cm})$ debido a la variabilidad espacial y relieve que presenta la ladera producto del pastoreo permanente.

La clase textural corresponde a franco a franco-arenoso. Cabe destacar que la pradera degradada (PD) se encuentra con alto grado de erosión de manto lo cual ha provocado afloramientos del horizonte $\mathrm{AC}$ con elevado porcentaje de arena $(>72 \%$, media) y mayor Da $1,3 \mathrm{~g} \mathrm{~cm}^{-3}$ en el perfil $0-25 \mathrm{~cm}$.

Bosque bajo pastoreo (BP) presenta un horizonte A difuso con $\mathrm{Da} 0,9 \mathrm{~g} \mathrm{~cm}^{-3} \mathrm{de} 0-5$ $\mathrm{cm}$; PS presenta valores de Da $1,0 \mathrm{~g} \mathrm{~cm}^{-3}$ $(5-15 \mathrm{~cm})$, con presencia del horizonte rico en arena en superficie; dado lo anterior, al considerar el perfil entre $0-25 \mathrm{~cm}$ en una hipótesis de degradación gradual de la unidad fisiográfica estudiada (ladera), se demues- 

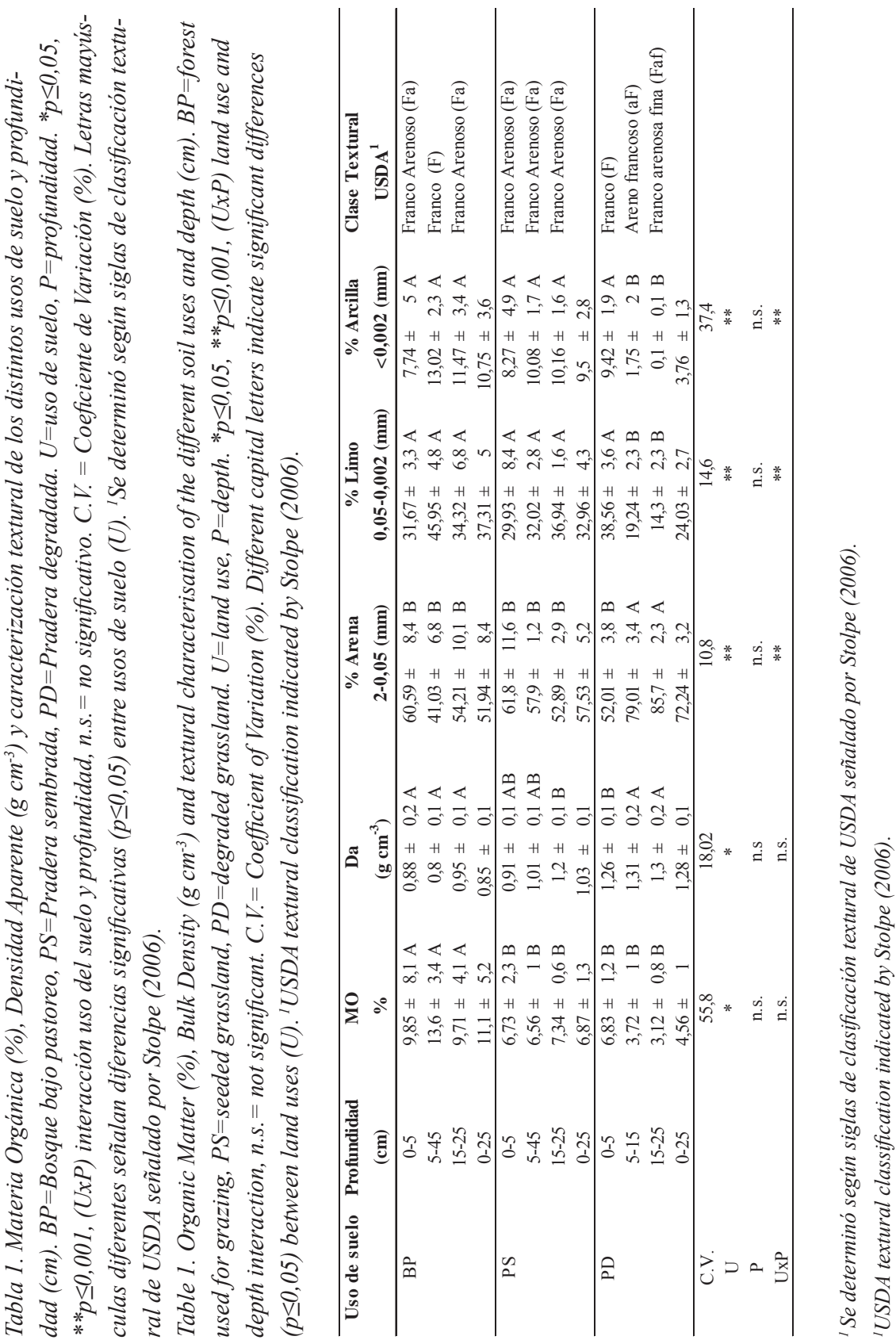
tra una sucesión desde un estado original a degradado desde BP a PS y PD respectivamente, con la disminución del contenido de materia orgánica en $59 \%(\mathrm{p} \leq 0,05)$, aumento de la densidad aparente en un 45,5\% $(p \leq 0,01)$, aumento en el contenido de arena en un $39 \%$ en profundidad $(\mathrm{p} \leq 0,01)$, disminución en el contenido de limo en $37 \%$ $(\mathrm{p} \leq 0,01)$ y arcilla $63 \%(\mathrm{p} \leq 0,01)$.

\section{Humedad aprovechable y porosidad}

Según Tabla 2 existieron diferencias significativas $(\mathrm{p} \leq 0,05)$ en la humedad aprovechable H.A. (\%C.C.-\%P.M.P.) entre los distintos usos de suelo (U); aun cuando el porcentaje de H.A. disminuye en profundidad un $31 \%$ entre $0-25 \mathrm{~cm}$, no existieron diferencias significativas $(p>0,05)$. La interacción entre uso y profundidad (UxP) fue significativa $(p \leq 0,05)$ en el tramo $0-5 \mathrm{~cm}$. H.A. según los distintos usos es considerada como baja a muy baja $(<10 \%)$, lo cual es característico de suelos arenosos y franco arenosos (Israelsen y Hansen, 1979).

Existieron variaciones en las distintas porosidades; el contenido de Macroporos varió significativamente $(\mathrm{p} \leq 0,05)$ entre los usos de suelo (U) e interacción con la profundidad (UxP) entre los 15 a $25 \mathrm{~cm}$ de profundidad entre PD y los otros usos (BP y PS). No existieron diferencias significativas en el contenido de Mesoporos en U y P; solo fue significativa la interacción (UxP) que ocurre de 15 a $25 \mathrm{~cm}$ de profundidad entre PS y PD. Existieron diferencias significativas en el contenido de Microporos entre los usos (U), sin significancia en profundidad (P) e interacción (UxP). La porosidad total (\%) se encuentra entre 51 a $67 \%$, lo cual es considerado como elevada (Sandoval et al., 2020).

\section{Agregados y estabilidad de agregados}

Según la Tabla 3, no existieron diferencias significativas $(\mathrm{p}>0,05)$ en DPM $(\mathrm{mm})$ entre los distintos usos de suelo (U), profundidades e interacción entre ambos factores; de igual forma, existen diferencias significativas en el IP $\left(\mathrm{g} \mathrm{kg}^{-1}\right)$ entre usos (U) e interacción (UxP), la cual se produce en 0-5 $\mathrm{cm}$ de profundidad.

Existieron diferencias significativas en el contenido de Macroagregados en los diferentes usos de suelo (U) no así en profundidad ni interacción (UxP); al respecto, varios autores señalan que los agregados del suelo se componen de Macroagregados y Microagregados agrupándose como estructuras aglomeradas de manera jerárquica y con una dinámica de ciclo de vida (formación y destrucción); este ciclo se inicia con la unión de partículas elementales derivando en Microagregados que luego se agrupan formando Macroagregados; los Macroagregados son más frágiles en su estructura que los Microagregados, y al ser disturbados se fragmentan en unidades menores (Microagregados) iniciando de esta manera un nuevo ciclo (Tisdal y Oades, 1982; Oades y Waters, 1991; Six et al., 1999); cabe indicar que suelos bajo formaciones vegetacionales permanentes, como praderas o bosques, tienden a presentar una mayor proporción de Macroagregados; por el contrario, suelos excesivamente labrados y que han perdido parte de su estructura, tiende a prevalecer un mayor contenido de Microagregados; dado esto, es deseable una mayor proporción de Macroagregados y una menor de Microagregados (Tisdall y Oades, 1979; Oades y Waters, 1991; Jastrow et al., 1998; Six et al., 1999; Bronick y Lal, 2005); al respecto Sandoval et al. (2020) indica que el contenido $\geq 50 \%$ de 


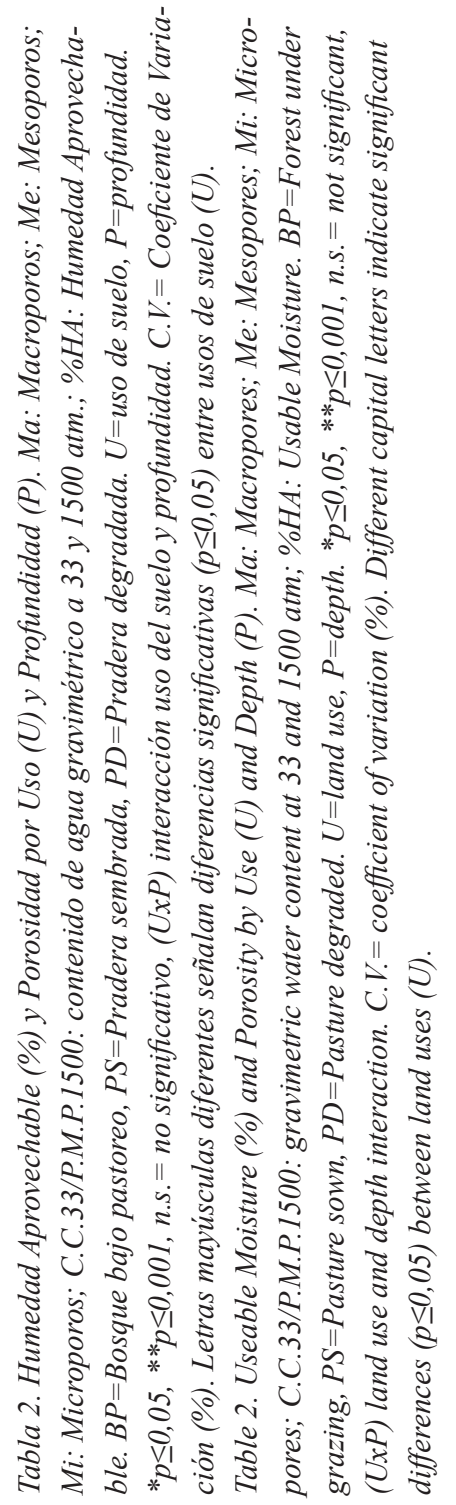

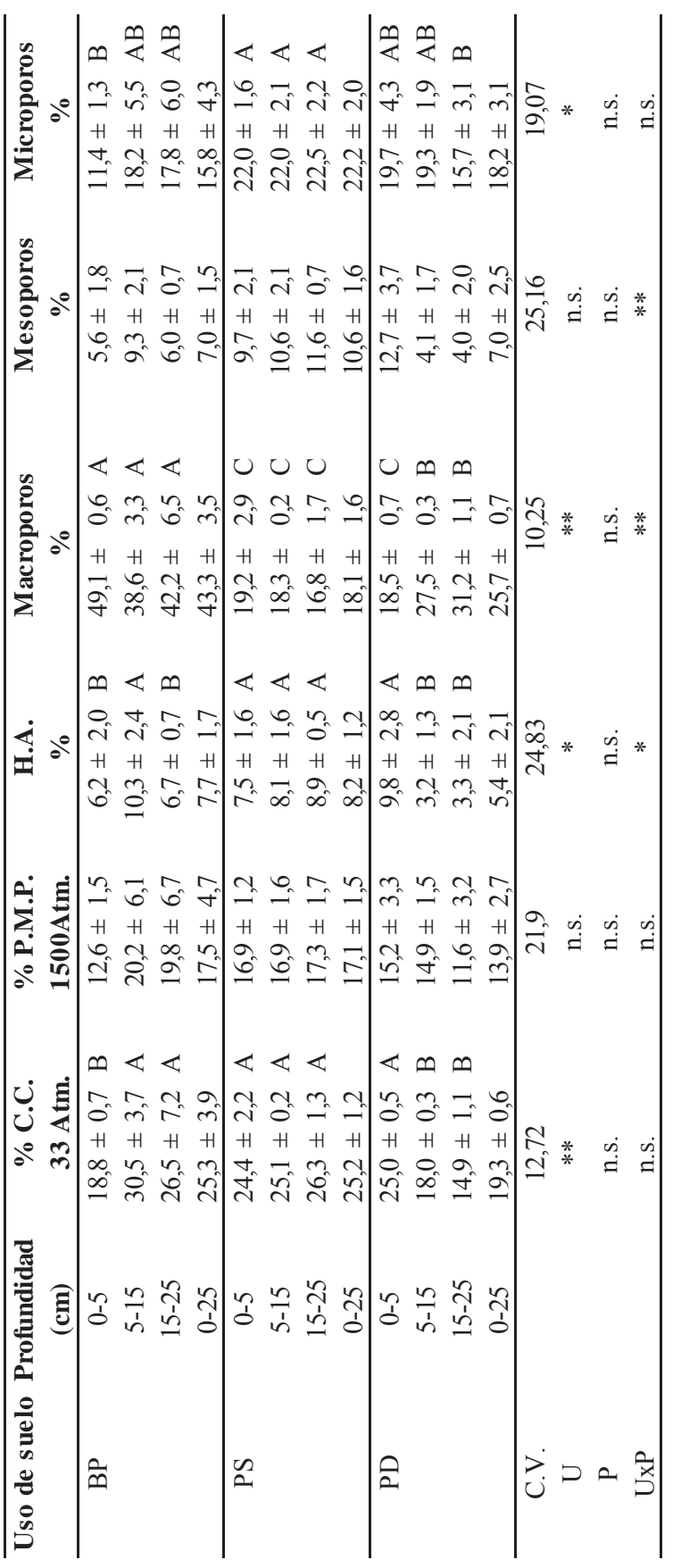

| Revista FAVE - Ciencias Agrarias 21 (1) 2022 
Macroagregados corresponde a una condi- dición favorable. El contenido de Microación favorable a muy favorable y $49-40 \%$ es una condición desfavorable; contrariamente, valores de Microagregados $\geq 50 \%$ corresponden a una condición desfavorable y $<40 \%$ muy favorable; bajo esta premisa, los resultados señalados en la Tabla 3 indican un contenido de Macroagregados en gregados es muy favorable; sin embargo, la categorización señalada está basada en el supuesto de proporcionalidad en cuanto a que un bajo contenido de Microagregados se compensaría con una proporción mayor de Macroagregados y viceversa lo cual no BP y PS como desfavorable y PD en con- traídos de los suelos estudiados.

Tabla 3. Estabilidad de agregados como Diámetro Peso Medio DPM, Índice de perdida y Macroagregados por uso de suelo y profundidad. DPM=Diámetro Peso Medio (mm). IP=Índice de Perdida $\left(\mathrm{g} \mathrm{kg}^{-1}\right) . B P=$ Bosque bajo pastoreo, $P S=$ Pradera sembrada, $P D=$ Pradera degradada. $U=$ uso de suelo, $P=$ profundidad. ${ }^{*} p \leq 0,05, * * p \leq 0,001$, n.s. $=$ no significativo entre usos de suelo $(U) .(U x P)$ interacción uso del suelo y profundidad. C.V.= Coeficiente de Variación (\%). Letras mayúsculas diferentes señalan diferencias significativas $(p \leq 0,05)$ entre usos de suelo (U).

Table 3. Aggregate stability as Mean Diameter Weight MDW, Loss Index and Macroaggregates by land use and depth. MDW= Mean Diameter Weight $(\mathrm{mm}) . I P=$ Loss Index $\left(\mathrm{g} \mathrm{kg}^{-1}\right) . B P=$ Forest used for grazing, $P S=$ Prairie sown, $P D=$ Prairie degraded. $U=$ land use, $P=$ depth. ${ }^{*} p \leq 0,05,{ }^{* *} p \leq 0,001$, $n . s .=$ not significant, $(U x P)$ land use and depth interaction. Different capital letters indicate significant differences $(p \leq 0,05)$ between land uses (U). C.V.= Coefficient of Variation (\%). Different capital letters indicate significant differences $(p \leq 0,05)$ between land uses $(U)$.

\begin{tabular}{|c|c|c|c|c|c|c|}
\hline \multirow{2}{*}{$\begin{array}{c}\text { Uso de suelo } \\
\text { BP }\end{array}$} & \multirow{2}{*}{$\begin{array}{c}\text { Profundidad } \\
\text { (cm) }\end{array}$} & \multirow{2}{*}{$\begin{array}{c}\begin{array}{c}\text { DPM } \\
\text { (mm) }\end{array} \\
0,21 \pm 0,1\end{array}$} & \multicolumn{2}{|l|}{$\begin{array}{c}\text { IP } \\
\left(\mathrm{g} \mathrm{kg}^{-1}\right)\end{array}$} & \multicolumn{2}{|c|}{$\begin{array}{l}\text { Macroagregados } \\
\%(\Sigma \geq 0,25 \mathrm{~mm} \emptyset)\end{array}$} \\
\hline & & & $207,6 \pm 4,8$ & B & $43,24 \pm 5,4$ & $\mathrm{AB}$ \\
\hline & 5-15 & $0,27 \pm 0,1$ & $353,0 \pm 9,8$ & A & $46,65 \pm 7,1$ & $\mathrm{AB}$ \\
\hline & $15-25$ & $0,27 \pm 0,1$ & $380,1 \pm 10,3$ & A & $47,01 \pm 10,8$ & $\mathrm{AB}$ \\
\hline & $0-25$ & $0,25 \pm 0,1$ & $313,6 \pm 8,3$ & & $45,63 \pm 7,8$ & \\
\hline \multirow[t]{4}{*}{ PS } & $0-5$ & $0,22 \pm 0,1$ & $258,8 \pm 6,2$ & A & $43,40 \pm 10,7$ & \\
\hline & $5-15$ & $0,21 \pm 0,1$ & $301,2 \pm 2,8$ & A & $40,56 \pm 0,6$ & B \\
\hline & $15-25$ & $0,19 \pm 0,1$ & $307,2 \pm 7,1$ & A & $40,15 \pm 6,2$ & B \\
\hline & $0-25$ & $0,20 \pm 0,1$ & $289,1 \pm 5,4$ & & $41,37 \pm 5,8$ & \\
\hline \multirow[t]{4}{*}{ PD } & $0-5$ & $0,22 \pm 0,1$ & $266,6 \pm 3,0$ & A & $44,73 \pm 5,1$ & $\mathrm{AB}$ \\
\hline & $5-15$ & $0,23 \pm 0,1$ & $183,2 \pm 3,8$ & B & $50,93 \pm 2,4$ & $\mathrm{AB}$ \\
\hline & $15-25$ & $0,26 \pm 0,1$ & $148,9 \pm 3,9$ & B & $57,60 \pm 7,2$ & $\mathrm{AB}$ \\
\hline & $0-25$ & $0,24 \pm 0,1$ & $199,6 \pm 3,6$ & & $51,09 \pm 4,9$ & \\
\hline C.V. & & 16,51 & 23,7 & & 16,46 & \\
\hline $\mathrm{U}$ & & n.s. & $*$ & & * & \\
\hline $\mathrm{P}$ & & n.s. & n.s. & & $\mathrm{n}$. & \\
\hline UxP & & n.s. & $*$ & & $\mathrm{n}$. & \\
\hline
\end{tabular}


Existieron diferencias significativas en el entre los distintos usos de suelo y profuncontenido de Microagregados tanto en uso $(\mathrm{U})$, profundidad $(\mathrm{P})$ e interacción $(\mathrm{UxP})$ didades (Tabla 3) presentan agregados muy inestables.

(Figura 2); la interacción se produce entre

El bosque bajo pastoreo (BP) está consBP y los otros usos entre 0-5 cm que es el es- tituido por especies dispersas del género trato más expuesto a la disgregación por ac- Nothofagus, en particular por Nothofagus ción del pastoreo y condiciones ambientales. antarctica que, por su condición de cadu-

Le Bissonnais (1996) señala que agre- cifolia, aporta hojarasca permanentemente gados $\leq 0,4 \mathrm{~mm}$ de DPM (mm) son muy (MO fresca) y que, en consecuencia, foinestables y se disgregan tendiendo a la for- menta una mayor actividad microbiana en mación sistemática de costras en el suelo; superficie contribuyendo a la formación y por su parte, Sandoval et al. (2020) señala estabilidad de agregados en el suelo; asiun nivel de estabilidad de agregados para mismo, especies del genero Nothofagus suelos minerales (Alfisol) con valores entre desarrollan asociaciones con hongos ecto0,5-0,7 de DPM ( $\mathrm{mm}$ ) se consideran inesta- micorrizicos que promueven la agregación bles y $<0,5$ como muy inestables; bajo esta y su estabilidad (Tisdall y Oades, 1982; consideración, todos los valores obtenidos Chaney et al., 1984; Gupta y Germida,

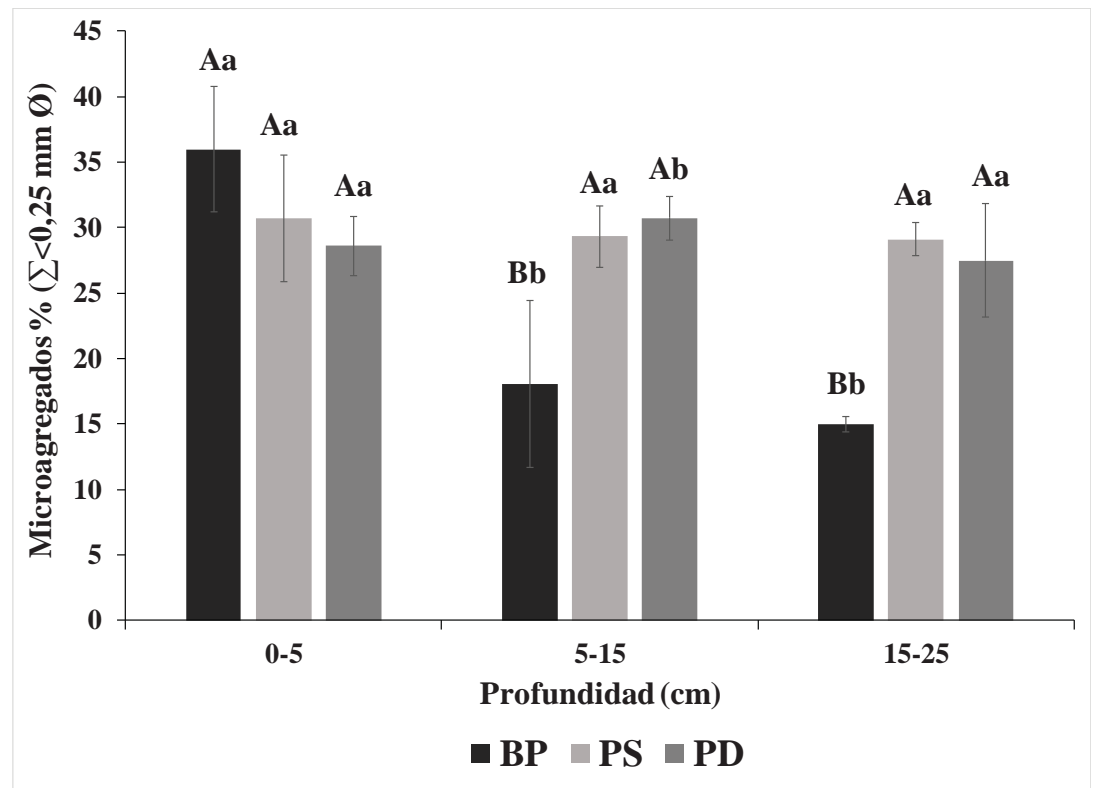

Figura 2. Microagregados por uso del suelo (U) y profundidad (P). Letras Mayúsculas corresponden a diferencias significativas $(p \leq 0,05)$ entre uso de suelo $(U)$. Letras Minúsculas corresponden a diferencias significativas $(p \leq 0,05)$ en profundidad $(P)$.

Figure 2. Microaggregates by land use $(U)$ and depth $(P)$. Capital letters correspond to significant differences $(p \leq 0,05)$ between land use $(U)$. Lowercase letters correspond to significant differences $(p \leq 0,05)$ in depth $(P)$. 
1988; Borie et al., 2000; García-Oliva et al., 2004; Alvear et al., 2007; Sandoval et al.,2010; Vial y Sandoval, 2015); por ende, se podría prever un efecto positivo por parte del estiércol aportado constantemente por el ganado que permanece bajo el dosel del bosque dado que mejora los indicadores de calidad física del suelo (Wortmann y Shapiro, 2008; Rayne y Aula, 2020); contrariamente a lo esperado, los valores obtenidos en BP de DPM (mm) fueron menores a 0,25 (mm) lo cual considerado como agregados muy inestables; esto se explicaría porque la degradación e integración de la MO al suelo es dependiente de la actividad biológica conjugadas con condiciones de temperatura y humedad (Göran et al., 2007) lo cual en la zona del estudio corresponde a una dinámica extremadamente lenta por las bajas temperaturas predominantes a lo largo del año y baja humedad relativa en superficie.

En relación a la pradera de alfalfa (PS), varios autores señalan que praderas permanentes, mixtas y con presencia de leguminosas, en particular alfalfa, promueven la formación de agregados estables y mejoran propiedades físicas como porosidad y conductividad hidráulica (Angers, 1992; Haynes y Beare, 1997; Rasse et al., 2000; Sandoval et al., 2011); sin embargo, el suelo de PS tiene un valor medio de $0,2 \mathrm{~mm}$ DPM considerado como muy inestable. Cabe señalar que el forraje de las praderas de alfalfa (PS) es utilizado como suplemento forrajero de invierno, por lo cual se excluye el ganado durante el crecimiento anual; dado esto, no hay aporte de MO producto de las deyecciones del ganado y procesos de reciclaje natural como ocurre con el pastoreo continuo de una pradera; se suma el efecto disgregante sobre el suelo de los sucesivos rastrajes en la etapa de establecimiento y el predominio de bajas temperaturas que ralentizan significativamente la dinámica biológica y por ende la restructuración del suelo (Shifang et al., 2008). Por su parte, la pradera degradada (PD) presentó valores de estabilidad de agregados considerados como inestables en base al bajo contenido de MO, baja cobertura vegetal, alto contenido de arena y degradación estructural generalizada en la que se encuentra.

Según CIREN (2010) el 78\% de la comuna de Lonquimay presenta un riesgo de erosión severa a muy severa, y en la actualidad $61 \%$ se encuentra con erosión activa en distinto grado; de igual forma, suelos cordilleranos en la Patagonia Argentina, con contenidos elevados de arena (69\%, franco arenoso, arenoso), bajos contenidos de materia orgánica y bajo pastoreo extensivo, registran tasas de sedimentos entre 144 y 750 $\mathrm{g} \mathrm{m}^{-2}$ bajo eventos de lluvia simulada de 50 mm en 30 minutos (La Manna et al., 2016); asimismo, alteraciones por uso del suelo como eliminación del bosque nativo, pastoreo recurrente y por largos periodos han alterado sus propiedades físicas acelerando su degradación y generando procesos erosivos severos (Dufilho et al., 2011; La Manna et al., 2018); cabe indicar, que la erosión altera la capacidad de retención y almacenamiento de agua repercutiendo en la capacidad de las plantas a colonizar, transformando la cobertura y la diversidad de especies, junto con otros efectos negativos como pérdida de materia orgánica, disgregación del suelo y perdida de nutrientes en los estratos superficiales, entre otros (Haynes y Francis, 1990; Reiners et al., 1994; Drewry et al., 2002; Geissen et al., 2009); dado lo anterior, se desprende que hay una serie de efectos aditivos, en temporalidad y magnitud, que configuran la degradación sostenida de los suelos de ladera estudiada, agravadas por su condición de uso. 


\section{CONCLUSIONES}

Se concluye que los suelos estudiados presentan condiciones generalizadas de degradación estructural, independiente a su uso y profundidad; esto ocurre debido al efecto aditivo de una serie de factores de degradación tales como un bajo contenido de materia orgánica, erosión, escasa estructura de los suelos, baja estabilidad de agregados y pastoreo excesivo que no permite recuperar la cubierta vegetal; a lo cual se suman condiciones climáticas con bajas temperaturas que ralentizan la dinámica biológica.

Por último, es altamente probable que, bajo la incidencia de eventos climáticos adversos, como lluvias intensas en corto tiempo y procesos de sequía prolongados, los suelos del sector incrementen su condición de degradación.

\section{BIBLIOGRAFÍA}

Alvear, M., Reyes, F., Morales, A., Arriagada, C. y Reyes, M. 2007. Actividad biológica y agregados estables al agua en dos tipos de formaciones vegetales de un bosque templado del centro - sur de Chile con perturbación antrópicas. Ecología Austral. 17.113-122.

Angers, D. A. 1992. Changes in soil aggregation and organic carbon under com and alfalfa. Soil Sci. Soc. Am. J.56, 1244-1249.

Blake, G.R.2008. Particle density. In: Chesworth W. (eds) Encyclopedia of Soil Science. Encyclopedia of Earth Sciences Series. Springer, Dordrecht. https://doi.org/10.1007/978-14020-3995-9 406

Borie, F., Rubio, R., Morales, A. y Castillo, C. 2000. Relación entre densidad de hifas de hongos micorrizógenos arbusculares y producción de glomalina con las características físicas y químicas de suelo bajo cero labranza. Revista Chilena de Historia Natural. 73, 749-756.

Bronick, C., and Lal, R. 2005. Soil structure and management: a review. Geoderma 124:3-22.

Chaney, K. and Swift, R.S., 1984. The influence of organic matter on aggregate stability in some British soils. J. Soil Science 35, 223-230.

CIREN, 2010. Determinación de la erosión actual y potencial de los suelos de Chile. Región de La Araucanía. CIREN. Síntesis de Resultados. Publicación N 149. 292 p.

Drewry, J., Lowe, J.A and Paton, J.R. 1999. Effect of sheep stocking intensity on soil physical properties and dry matter production on a Pallic Soil in Southland, New Zealand. Journal of Agricultural Research. 42(4), 493-499.

Dufilho, A., Frugoni, M., Macchi, P., \& Thot, A. 2011. Estimación de Erosión Hídrica de Suelos para plan de Manejo del Campo Forestal Aguas Frías, Patagonia Argentina. Boletín Geográfico, 0(33), 69-85. En: http://revele. uncoma.edu.ar/htdoc/revele/index.php/geografia/article/view/73

FIA, 2017. Atlas Agroclimático de Chile. Estado actual y tendencias del clima. Tomo IV. Regiones del Biobío y de La Araucanía. Proyecto Centro AGRIMED, Facultad de Ciencias Agronómicas de la Universidad de Chile FIA. 140 p.

Fuentes-Ramírez, A., Pauchard, A., Hauenstein, E. 2011. Composición de la flora de praderas andinas en la Reserva Nacional Alto Bío-Bío (Lonquimay-Chile) y su relación con el régimen de pastoreo. Gayana Bot.68(1): 28-39.

García-Oliva, F., Oliva, M. and Sveshtarova, B. 2004. Effect of soil macroaggregates crusing on $\mathrm{C}$ mineralization in tropical deciduous forest ecosystem. Plant and Soil, 259: 297-305.

Geissen, V., Sanchez-Hernandez, R., Kampichler, C., Ramos-Reyes, R., Sepúlveda-Lozada, A. and Ochoa-Goana, S. 2009. Effects 
of land-use change on some properties of tropical soils - An example from Southeast Mexico. Geoderma, 151(3-4), 87-97.

Göran I. Ågren, J.Å. Martin Wetterstedt, What determines the temperature response of soil organic matter decomposition?, Soil Biology and Biochemistry, Volume 39, Issue 7, 2007, Pages 1794-1798, https://doi.org/10.1016/j. soilbio.2007.02.007.

Gupta, U. and J. Germida. 1988. Distribution of microbial biomass and its activity in different soil aggregate size classes as affected by cultivation. Soil Biol. Biochem. 20: 777-786.

Hao, X.; Ball, C.; Culley, J.; Carter, M.; Parking, G. 2019. Soil Density and Porosity. Lethbridge, Agri-Food Canada. Chapter 57. 753-759.

Hauenstein, E., Palma, R., González, M., \& Ahumada, M. 2003. Composición florística de praderas alto andinas de la zona de Lonquimay (IX Región, Chile). Agro Sur, 31(1), 8-20. doi:10.4206/agrosur. 2003.v31n1-02

Haynes, R.J. and Beare, M.H. 1997. Influence of six crop species on aggregate stability and some labile organic matter fractions. Soil Biol. Biochem. 29 (1), 1647-1653.

Haynes, R.J. and Francis G.S. 1990. Effects of mixed cropping farming systems on changes in soil properties on the Canterbury plains. New Zeland Journal of Ecology. 14, 73-82.

Israelsen, O. W.; Hansen, V. E. Principios y aplicaciones del riego. 2.ed. Barcelona: Editorial Reverte, 1965. 400p.

Jastrow, J.D., Miller, R.M., Lussenhop, J. 1998. Contributions of interacting biological mechanisms to soil aggregate stabilization in restored prairie. Soil Biol. Biochem. 30(7), 905-916.

Kemper, W. and Rosenau R. 1986. Aggregate stability and size distribution. In, Klute, A. (ed). Methods of Soil Analysis, Part I. Agronomy Monographs, 9. 425-442.

La Manna, L.; Buduba, C. and Rostagno, C.2016. Soil erodibility and quality of vol- canic soils as affected by pine plantations in degraded rangelands of NW Patagonia. European Journal of Forest Research. 135. Doi: 10.1007/s10342-016-0961-z.

La Manna, L.; Gaspar, L.; Rostagno, C., Laura Quijano, L. and Navas, A. 2018. Soil changes associated with land use in volcanic soils of Patagonia developed on dynamic landscapes. CATENA, 166,229-239. Doi: 10.1016/j.catena.2018.03.025.

Le Bissonnais, Y. 1996. Aggregate stability and assessment of soil crustability and erodibility, I. Theory and methodology. Eur. J. Soil Sci., 47, 425-437.

Luzio, W., Seguel y O. Casanova, M. 2009. Suelos de la Zona Húmeda (Desde $43^{\circ}$ LS hasta 50 LS), 263-289. En, Suelos de Chile (Luzio, W., Editor). Universidad de Chile.

Luzio, W.; Norambuena, P.; Avendaño, P.; Muñoz, F.; Torres, M.; Sandoval, G. y Muñoz F. 2013. Estudio de aguas y suelos en valles cordilleranos con población indígena. Regiones del Biobío y La Araucanía. Chile. Corporación Nacional de Desarrollo Indígena y EDAFICA. 125 p.

Murty, D., Kirschbaum, M. U. F., McMurtrie, R. E. and McGilvray, A. 2002. Does conversion of forest to agricultural land change soil carbon and nitrogen? a review of the literature. Review. Global Change Biology, 8(2), 105-123.

Oades, J.M. and Waters, A.G. 1991. Aggregate hierarchy in soils. Aust. J. Soil Res. 29,815828.

Rasse, D.P., Smucker, A.J.M. and Santos, D. 2000. Alfalfa Root and Shoot Mulching Effects on Soil Hydraulic Properties and Aggregation. Soil Sci. Soc. Am. J., 64: 725-731. doi:10.2136/sssaj2000.642725x

Rayne, Natasha, and Lawrence Aula. 2020. Livestock Manure and the Impacts on Soil Health: A Review. Soil Systems 4, no. 4: 64. https://doi.org/10.3390/soilsystems4040064 
Reiners W.A., Bouwman A.F., Parsons W.F., Keller, M. 1994. Tropical Rain Forest Conversion to Pasture, Changes in Vegetation and Soil Properties. Ecological Applications. 4(2), 363-377.

Rodríguez, A., Arbelo C., Guerra J., Mora, J. Notario J and Armas C.2006.Organic carbon stocks and soil erodibility in Canary Islands Andosols. CATENA, 66(3), 228-235.

Sadzawka, A., Carrasco, M., Grez, R., Mora, M., Flores, H., Neaman, A., 2006. Métodos de análisis recomendados para los suelos de Chile. Serie Actas INIA $N^{\circ} 34$. Instituto de Investigaciones Agropecuarias, Santiago, Chile. 164 p.

Salas, R. y Cabalceta G. 2009. Manejo del Sistema Suelo - Pasto: partida para la producción de forrajes. Centro de Investigaciones Agronómicas, Universidad de Costa Rica. Producción de forrajes: clave para el éxito de la lechería. Conferencia. Congreso Nacional Lechero. 59 p.

Sandoval E., M., Peréz Q., Recio., Sánchez-Hernández., Capulín A. 2020. Desarrollo de un manual de evaluación y restauración agro-ecológica de suelos de uso agropecuarios afectados por incendios. Proyecto ARIII70003. Agencia Nacional de Investigación y Desarrollo (ANID). 80 p.

Sandoval, M., Celis, J. and Morales, P. 2011. Structural remediation of an Alfisol by means of sewage sludge amendments in association with yellow serradela (Ornithophus compressus L.). J.Soil Sci. Plant Nutr., 11(1), 68-78.

Sandoval, M., Celis, J., Stolpe, N., y Capulín, J. 2010. Efecto de enmiendas con lodos urbanos y de salmonicultura en la estructura de un Entisol y un Alfisol en Chile. Agrociencia, 44(5), 503-515.

Sandoval, M., Dörner J., Seguel S., Cuevas, J. y Rivera, D. 2012. Métodos de análisis físicos de suelos. Universidad de Concepción. Publicaciones Departamento de Suelos y Recursos Naturales, Chillán, Chile, $\mathrm{N}^{\circ} 50.80$ p.
Schlatter, J.; Grez, R. y Gerding, V. 2003. Manual para el reconocimiento de suelos. $3 \mathrm{a}$ Ed. Universidad Austral de Chile, Valdivia, Chile. $123 \mathrm{p}$.

Stolpe, N. 2006. Descripciones de los principales suelos de la VIII Región de Chile. Departamento de Suelos y Recursos Naturales. Ediciones Universidad de Concepción. 1(1). 84 p.

Shifang, P., Hua, F., Changgui, W. 2008. Changes in soil properties and vegetation following exclosure and grazing in degraded Alxa desert steppe of Inner Mongolia, China. Agriculture, Ecosystems \& Environment, 124 (1-2), 33-39.

Six, J., Elliott, E.T. and Paustian, K. 1999. Aggregate and soil organic matter dynamic under conventional and no-tillage systems. Soil Sci. Am. J. 63,1350-1358.

Tisdall, J.M. and Oades, J.M. 1982. Organic matter and water-stable aggregates in soils. Journal of Soil Science, 33,141-163.

Tisdall, J.M. and Oades, J.M. 1979. Stabilization of soil aggregates by the root systems of ryegrass. Aust. J. Res. 17,429-441.

Tosso T., J.1985. Suelos volcánicos de Chile [en línea]. Santiago. En: https://biblioteca.inia. $\mathrm{cl} / \mathrm{handle} / 123456789 / 35623$

Universidad de Chile. 2012. Estado del arte de modelos para la investigación del cambio global. Departamento de Geofísica, Facultad de Ciencias Físicas y Matemáticas, Universidad de Chile, Santiago, Chile.32 p.

Vial, M. and Sandoval, M. 2015. Soil structural condition and its relationship with pastures under different conditions in the Simpson Valley (Humid western Patagonia, Chile). Idesia (Arica), 33(4), 31-40. https://dx.doi. org/10.4067/S0718-34292015000400005

Wortmann, C.S., Shapiro, C.A. 2008.The effects of manure application on soil aggregation. Nutr Cycl Agroecosyst 80, 173-180. https:// doi.org/10.1007/s10705-007-9130-6 